MATEC Web of Conferences 50, 02004 (2016)

DOI: $10.1051 /$ matecconf/20165002004

(c) Owned by the authors, published by EDP Sciences, 2016

\title{
A Simple Short Circuit Analysis for Power Networks
}

\author{
İlhan Koşalay \\ Ankara Univ, Faculty of Engineering, Electrical and Electronics Engineering Department Ankara, Turkey
}

\begin{abstract}
This study investigates the transient behavior of short circuits in power circuits. The circuit consists of two part; input part and load part. These two parts are connected with a circuit breaker switch. The circuit works in two modes; first mode is when the switch is open and second mode is when the switch is closed. This study analyses the circuit when the switch is closed. The analysis is done with different types of closing angle. The analysis is done by forming state equations and those equations are solved numerically by using Matlab. The analysis and conclusion is performed by observing the behaviors of the graphs.
\end{abstract}

\section{Introduction}

Closing operation of a transmission line or a inductive / capacitive circuit can produce transient overvoltage's due to several factors. One of the most important factors, that have more influence on the peak value of voltage, can be assumed as the instant of closing [1]. For the transmission lines, the transient over voltages due to the switching of circuit are limited because of pre insertion resistors in the line circuit breakers $[2,3]$. On the other side, using controlled closing can effectively limit the overvoltages and eliminate the need for closing resistors [4]. For an ideal condition, the circuit breaker (CB) contacts should touch completely when the voltage over the contacts is zero. But in real applications, the $\mathrm{CB}$ operating time may change. The variation of closing time of the $\mathrm{CB}$ contacts is assumed to change as a Gaussian distribution [5]. The value of the rate of decrease of dielectric strength should exceed the maximum derivative of the applied voltage for proper operation of $\mathrm{CB}$. The maximum pre-striking voltage is determined according to the statistical scatter of the contact's speed [6].

The purpose of this paper is to create a simple and effective tool for investigation voltage changes due to the closing operations. The newly developed tool for closing transients is in good agreement with the practical applications. Inductive / capacitive circuit transient over voltages were examined in [7]. In this paper, a simulation analysis based on state equations is presented to find the closing transient over voltages and the other possible voltage variations due to the short circuits.

\section{State Model For The Single Phase Switch Opening And Closing}

Figure 1 shows and proposes a circuit model for switching analysis for the power circuits.

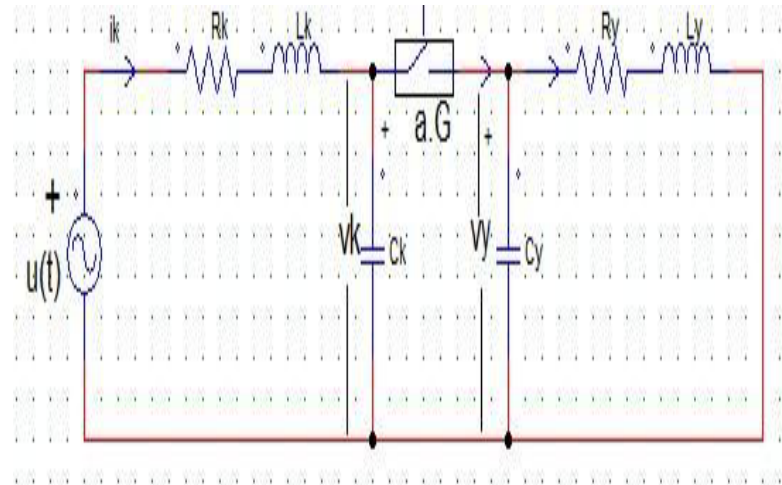

Figure 1. A proposed switching transient investigation model

If the switch is open $a=0 ; \quad a \cdot G=0$ and it means no conductivity. When the switch is closed $a=1$; a.G is very large, it means that circuit is closed. Source voltage is assumed pure sinus signal as $\mathrm{U}(\mathrm{t})=\sqrt{2} \mathrm{~V} \cdot \sin (\omega t+\theta)$

State model of the system is derived the circuit which is shown in figure 1 and they are given in below,

$$
\begin{aligned}
& L_{k} \cdot \frac{d i_{k}}{d t}=-R_{k} i_{k}-v_{k}-\mathrm{u}(\mathrm{t}) \\
& C_{k} \cdot \frac{d v_{k}}{d t}=i_{k}-\mathrm{a} \cdot \mathrm{G}\left(v_{k}-v_{y}\right) \\
& L_{y} \cdot \frac{a l_{y}}{d t}=-R_{y} i_{y}+v_{y} \\
& C_{y} \cdot \frac{d v_{y}}{d t}=a \cdot G\left(v_{k}-v_{y}\right)-i_{y}
\end{aligned}
$$

As a result, state equations can be summarized as below, 
$\frac{d}{d t}\left[\begin{array}{c}i_{k} \\ v_{k} \\ i_{y} \\ v_{y}\end{array}\right]=\left[\begin{array}{cccc}-\frac{R_{k}}{L_{k}} & -\frac{1}{L_{k}} & 0 & 0 \\ \frac{1}{c_{k}} & -\frac{a \cdot G}{c_{k}} & 0 & \frac{a_{G} G}{c_{k}} \\ 0 & 0 & -\frac{R_{y}}{L_{y}} & \frac{1}{L_{y}} \\ 0 & \frac{a_{\cdot} G}{c_{y}} & -\frac{1}{c_{y}} & -\frac{a \cdot G}{c_{y}}\end{array}\right]\left[\begin{array}{c}i_{k} \\ v_{k} \\ i_{y} \\ v_{y}\end{array}\right]+\left[\begin{array}{c}-\frac{1}{L_{k}} \\ 0 \\ 0 \\ 0\end{array}\right] u(t)$

Output magnitudes of the system are mentioned in equation (2.6). Here; $i_{A}$ : Current passing through the switch and $u_{\tau}$ : Recovery voltage

$\left[\begin{array}{c}i_{A} \\ u_{\tau}\end{array}\right]=\left[\begin{array}{cccc}0 & a . G & 0 & -a . G \\ 0 & 1 & 0 & -1\end{array}\right]\left[\begin{array}{c}i_{k} \\ v_{k} \\ i_{y} \\ v_{y}\end{array}\right]$

When the switch is closed $\mathrm{a}=1$

$u_{k}=v_{y} ; \frac{d v_{k}}{d t}=\frac{d v_{y}}{d t}$

a.G: Very large,

In this situation both $u_{k}$ and $v_{y}$ can not be state variables at the same time. If we $v_{y}$ is chosen as a state variable equation (2.2) and 2.(4) can be written as shown below:

$$
\begin{aligned}
& C_{k} \cdot \frac{d v_{y}}{d t}=i_{k} \\
& C_{y} \cdot \frac{d v_{y}}{d t}=\mathrm{i}_{\mathrm{y}}
\end{aligned}
$$

Adding equation (7) and (8) gives;

$$
\begin{aligned}
& \left(C_{k}+C_{y}\right) \cdot \frac{a v_{y}}{d t}=i_{k}-i_{y} \\
& \frac{a v_{y}}{d t}=\frac{1}{c_{k}+c_{y}} i_{k}-\frac{1}{c_{k}+c_{y}} i_{y}
\end{aligned}
$$

For the closed switch condition, state equations become as below:

$\frac{d}{d t}\left[\begin{array}{l}i_{k} \\ i_{y} \\ v_{y}\end{array}\right]=\left[\begin{array}{ccc}-\frac{R_{k}}{L_{k}} & 0 & -\frac{1}{L_{k}} \\ 0 & -\frac{R_{y}}{L_{y}} & \frac{1}{L_{y}} \\ \frac{1}{c_{k}+c_{y}} & \frac{1}{c_{k}+c_{y}} & 0\end{array}\right]\left[\begin{array}{l}i_{k} \\ i_{y} \\ v_{y}\end{array}\right]+\left[\begin{array}{c}-\frac{1}{L_{k}} \\ 0 \\ 0\end{array}\right] u(t)$

$i_{A}=\left[\begin{array}{lll}\frac{c_{y}}{c_{k}+c_{y}} & \frac{c_{k}}{c_{k}+c_{y}} & 0\end{array}\right]\left[\begin{array}{l}i_{k} \\ i_{y} \\ v_{y}\end{array}\right]$

When the switch is closed, it can be investigated the below transient events using equation (11) and (12):

a) Energization of large inductive load: $C_{k}$ and $C_{y}$ are negligible

b) Energization of large capacitive load: $R_{y}$ and $L_{y}$ are negligible. c) Short circuit current: $R_{y}=0 ; C_{k}, C_{y}$ and $L_{y}$ are negligible.

From special cases $\mathrm{a}, \mathrm{b}$, and $\mathrm{c}$ above first or second order differential equation is obtained and analytical solution of the equation can be found. Since the circuit was operating in continuous in sinusoidal mode before the switch was closed, in the investigation of transient events when the switch is closed initial conditions is obtained as follows (Here, $\theta$ : Respective angle when the switch is closed.).

$\left|Z_{k}\right|=\sqrt{R_{k}^{2}+\left(\omega L_{k}-\frac{1}{\omega c_{k}}\right)^{2}} ; \quad \alpha=\tan ^{-1}\left(\frac{\omega^{2} L_{k} c_{k}-1}{\omega R_{k} c_{k}}\right) ;$

$i_{k}(0)=\frac{\sqrt{2} V}{\left|z_{k}\right|} \sin (\theta-\alpha)$

$v_{k}(0)=\frac{\sqrt{2} V}{\omega c_{k}\left|z_{k}\right|} \sin \left(\theta-\alpha-90^{\circ}\right)=v_{y}(0)$

$i_{y}(0)=\mathbf{0}$

A graphical based interface is created in MATLAB environment is shown in figure 2 .

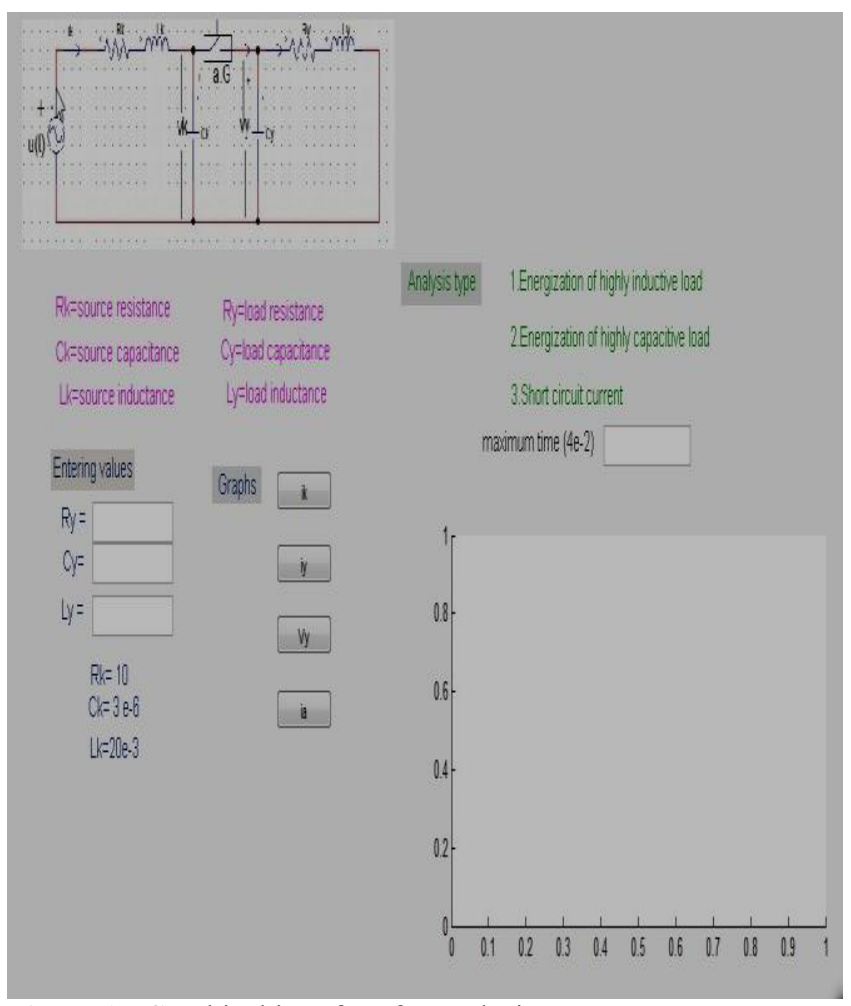

Figure 2. Graphical interface for analysis.

\section{Analysis of Short Circuit}

Short circuit is analyzed by letting the value of load resistance $\mathrm{Ry}=0$ with large value of $C y(0.001 \mathrm{~F})$ 


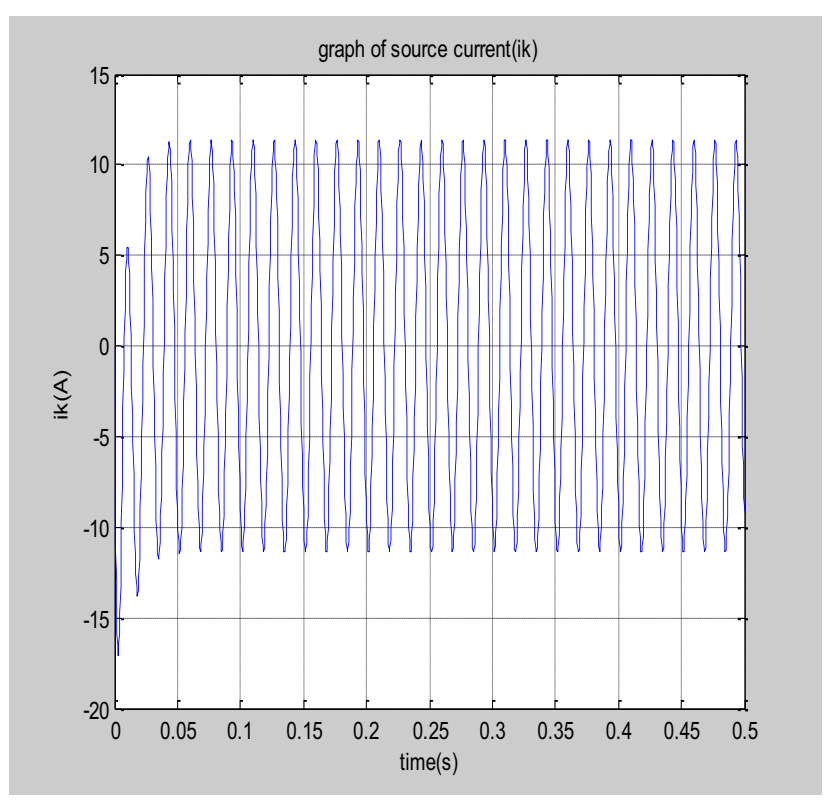

Figure 3. Graph of the source current during energization of short circuited load for $\theta=\pi / 2$.

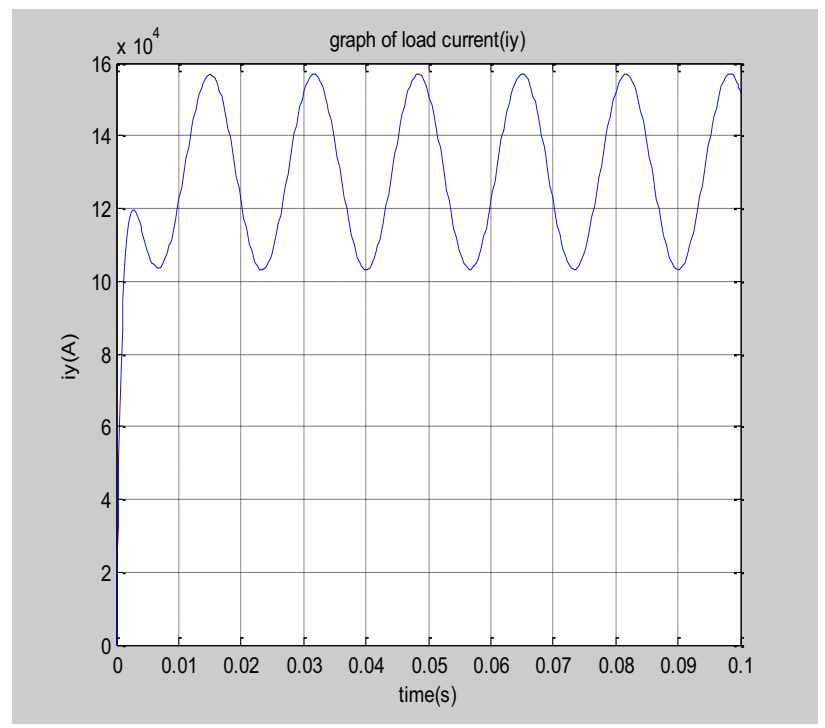

Figure 4. graph of the load current during energization of short circuited load for $\theta=\pi / 2$.

Figure 3-6 show different outputs related to short circuit condition respectively. All values of $\theta$ makes the load to show transient behavior at the beginning, but for different values the level at which current attains steady state is different. Small values of $\theta$ causes the current to reach its steady state at lower values while $\theta=\pi / 2$ gives the highest level.

The time spent before reaching state is same for different values of $\theta$, for the graphs above it shows that every graph reaches its steady state at 0.01 second.

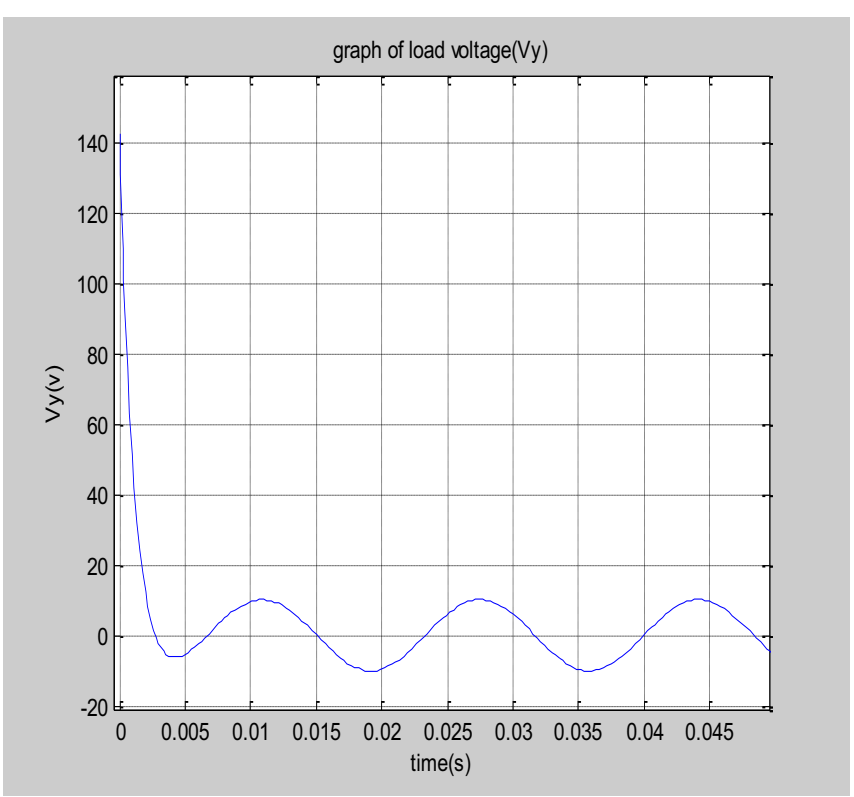

Figure 5. Graph of the load voltage during energization of short circuited load for $\theta=\pi / 2$

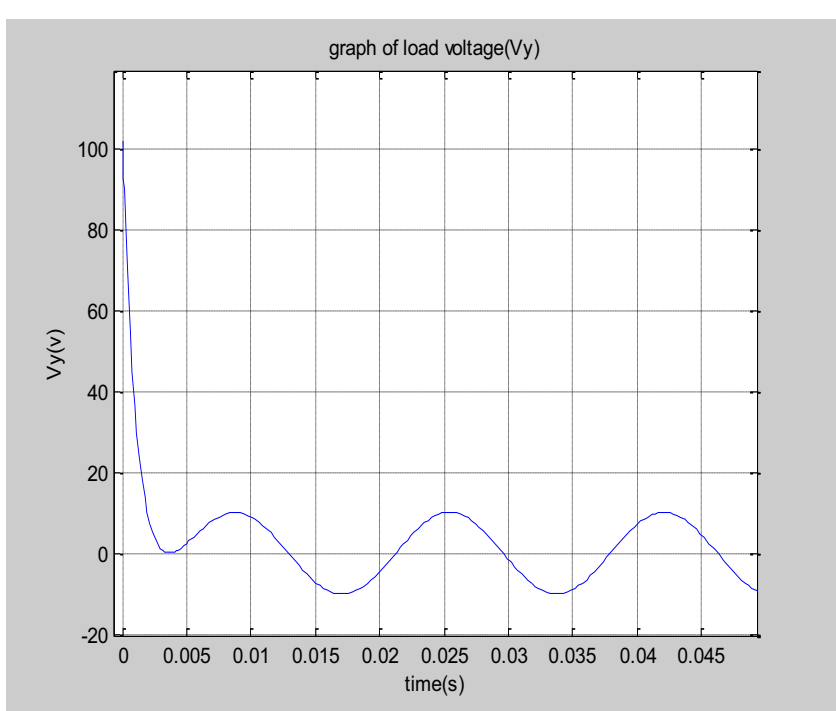

Figure 6. graph of the load voltage during energization of short circuited load for $\theta=3 \pi / 4$.

The load voltage starts at its initial value and falls rapidly. After some times it attains its steady state, for different values of $\theta$ the steady state peak value is same but the initial values are different. Value $\theta=\pi / 2$ gives the highest initial value.

\section{Conclusions}

When the short circuit occurs in power networks, the short circuit makes the load to show transient behavior. The transient behaviors are different for different types of loads. Depending on values of angle when the circuit is closed $(\theta)$, transient time can be short or long. During transient periods, overvoltage occurs most of the time. To analyze and examine this kind of overvoltage is not easy 
every time due to complex parameters. This study analyzed closing transients in case of short circuit.

\section{References}

1. J. A. Martinez-Velasco, Power System Transients, Parameter Determination. Boca Raton, FL: CRC, Taylor \& Francis Group, 2010.

2. P. Mestas and M. C. Tavares, "Comparative analysis of control switching transient techniques in transmission lines energization maneuver," presented at the Int. Conf. Power Syst. Transients, Lyon, France, Jun. 4-7, 2007.

3. M. Sanaye-Pasand, M. R. Dadashzadeh, and M. Khodayar, "Limitation of transmission line switching overvoltages using switchsync relays," presented at the Int. Conf. Power Syst. Transients, Montreal, QC, Canada, Jun. 19-23, 2005.

4. K. M. C. Dantas,D. Jr. Fernandes, W. L. A. Neves, B. A. Souza, and L. C. A. Fonseca, "Mitigation of switching overvoltages in transmission lines via controlled switching," presented at the IEEE Power Energy Soc. Gen. Meeting, Pittsburgh, PA, Jul. 20-24, 2008.

5. U. Samitz, H. Siguerdidjane, F. Boudaoud, P. Bastard, J. P. Dupraz,M. Collet, J.Martin, and T. Jung, "On controlled switching of high voltage unloaded transmission lines," in Proc. Elektrotech. Inf., Paris, France, Aug. 2002, vol. 119 , no. 12, pp. 415-421.

6. K. Frohlish, C. Hoelzl, M. Stanek, A. C. Carvalho, W. Hofbauer, P. Hoegg, B. L. Avent, D. F. Peelo, and J. H. Sawada, "Controlled closing on shunt reactor compensated transmission lines part I: Closing control device development," IEEE Trans. Power Del., vol. 12, no. 2, pp.734-740, Apr. 1997.

7. MBONDE Shaban, KOŞALAY İlhan, "State equation based closing simulation", Communications, Series A2-A3Physics, vol: 57, number: 2 , page: 013-023, ISSN: 1303-6009, 2015 